\title{
Association of Circulating TXNIP Levels with Fatty Liver in Newly Diagnosed Type 2 Diabetes Mellitus
}

\author{
Yuting Guo',* \\ Juan Chen (iD) $2, *$ \\ Nan $\mathrm{Liu}^{3}$ \\ Zheng Liu ${ }^{4}$ \\ Bimin $\mathrm{Shi}^{4}$ \\ Hong Sun (iD) 4 \\ 'Department of Endocrinology and \\ Metabolism, Affiliated Kunshan Hospital \\ of Jiangsu University, Kunshan, Jiangsu, \\ People's Republic of China; ${ }^{2}$ Department \\ of Endocrinology, Jiangsu Province \\ Hospital of Chinese Medicine, Affiliated \\ Hospital of Nanjing University of Chinese \\ Medicine, Nanjing, People's Republic of \\ China; ${ }^{3}$ Department of Radiology, The \\ First Affiliated Hospital of Soochow \\ University, Suzhou, Jiangsu, People's \\ Republic of China; ${ }^{4}$ Department of \\ Endocrinology and Metabolism, The First \\ Affiliated Hospital of Soochow University, \\ Suzhou, Jiangsu, 2I5006, People's \\ Republic of China \\ *These authors contributed equally to \\ this work
}

Background and Aims: Thioredoxin-interacting protein (TXNIP), a crucial modulator of the redox system, plays a crucial role in modulating lipid/glucose metabolism. Hence, this study aimed to explore whether circulating TXNIP is associated with non-alcoholic fatty liver disease (NAFLD) among patients with type 2 diabetes mellitus (T2DM).

Methods: We enrolled 110 new patients with T2DM. In this study, we determined hepatic fat fraction (HFF), which represents a hepatic fat level, by Dixon magnetic resonance imaging. TXNIP and the other biochemical profiles of the patients were measured using fasting plasma.

Results: Among the 110 patients with T2DM, 41 were classified as without fatty liver, whereas 34 and 35 were with mild and moderate-to-severe fatty liver, respectively. The patients with diabetes and advanced fatty liver had significantly higher TXNIP levels $(P<0.001)$ than other patients. The prevalence of severe NAFLD showed an increasing trend with the increase in TXNIP quartiles (for all trends, $P<0.05$ ). HFF showed a positive correlation with TXNIP $(\mathrm{r}=0.516, P<0.001)$. Even main risk factors were adjusted, TXNIP level was associated with NAFLD as analyzed by logistic regression.

Conclusion: TXNIP level remarkably increases among diabetics, which shows its positive relationship with the severity of NAFLD. TXNIP is a promising NAFLD biomarker that offers an efficient way to evaluate and monitor fatty liver progression among patients with T2DM.

Keywords: thioredoxin-interacting protein, non-alcoholic fatty liver disease, type 2 diabetes mellitus

\section{Introduction}

Non-alcoholic fatty liver disease (NAFLD), a frequently occurring and potentially serious condition, is often closely linked to metabolic syndrome and diabetes mellitus; it affects more than $25 \%$ of adults and $60 \%$ of patients with diabetes worldwide. ${ }^{1}$ NAFLD includes various clinical manifestations, such as the accumulation of liver fat. Its symptoms include plain liver steatosis, non-alcoholic steatohepatitis (NASH, a severe type), and eventually advanced fibrosis that results in hepatocellular carcinoma and cirrhosis. Many studies support rapidly rising rates of the aforementioned pathological liver conditions among patients with type 2 diabetes mellitus (T2DM). ${ }^{2}$ Furthermore, for patients with T2DM, NAFLD may predict severe dyslipidemia and insulin resistance (IR) in hepatic tissues than in those without NAFLD. ${ }^{3}$ Hence, focusing on patients with diabetes who suffer from NAFLD is particularly important. Although the pathogenesis of fatty liver is highly complicated, IR, inflammation, and oxidative stress play critical roles in NAFLD occurrence and progression. ${ }^{4}$ Initially,
Correspondence: Hong Sun Email sunhong_61I@I26.com 
the double-hit hypothesis ${ }^{5}$ stated that IR-mediated hepatocellular lipid accumulation represents the first hit and is succeeded by the second hit wherein oxidative stress induces hepatocellular inflammation, thus promoting a progression towards NASH. As reported by Moschen and Tilg in 2010, multiple hits that acted parallelly performed well in explaining fatty liver genesis and progression. These multiple hits include lipid peroxidation, oxidative stress, inflammation, and mitochondrial dysfunction that can cause hepatocyte injury and liver damage. ${ }^{6}$

In the last few years, a stress-response gene encoding thioredoxin-interacting protein (TXNIP) has emerged as a crucial modulator of the redox system, which affects the cellular redox balance by suppressing thioredoxin. Recently, evidence has shown that TXNIP affects metabolic regulation and is associated with metabolic disorders such as hypertension, T2DM, and NAFLD. ${ }^{7-9}$ TXNIP expression is strongly upregulated in human diabetes, which has a critical role in diabetic complication genesis and progression. ${ }^{10,11}$ Clinical studies have reported that serum TXNIP is associated with diabetic nephropathy, diabetic retinopathy, diabetic peripheral neuropathy, and arteriosclerosis in patients with diabetes. ${ }^{12,13}$ However, whether circulating TXNIP is related to NAFLD in patients with T2DM remains unknown. Recently, Park et al reported increased TXNIP in liver biopsies of patients with NAFLD that contributed to the generation of fatty liver. ${ }^{14}$ Further, animal studies showed that TXNIP overexpression causes hepatic lipid accumulation and inflammation in rats fed with a high-fructose diet. ${ }^{15}$ Moreover, TXNIP deletion ameliorates hepatic steatosis and inflammatory response in a mouse model of NASH. ${ }^{16}$ These results suggested that TXNIP may be a critical regulating factor for the metabolism of hepatic lipid. Based on the aforementioned studies, we tested circulating TXNIP levels and evaluated their association with liver fat content among patients with T2DM in this study. Our study aimed to determine whether circulating TXNIP was associated with hepatic fat content and could be used as a serum biomarker of fatty liver among patients with T2DM.

\section{Methods}

\section{Subjects and Design}

The present cross-sectional study was approved by the Ethics Committee of First Affiliated Hospital of Soochow University per the principles of the Helsinki Declaration. Between January 2017 and December 2018, a total of 110 patients who were newly diagnosed with T2DM were recruited by the Department of Endocrinology and Metabolism of First Affiliated Hospital of Soochow University. All participants provided informed consent for participation. The diagnosis of T2DM was based on the 1999 World Health Organization criteria. Patients with T2DM conforming to the following criteria were enrolled: age $\geq 18$ years and had not received any diabetes treatment. NAFLD was diagnosed according to "Non-alcoholic Fatty Liver Disease Diagnosis and Treatment Guidelines." Each subject underwent quantitative liver magnetic resonance imaging (MRI) using the $1.5 \mathrm{~T}$ whole-body human MRI scanner by the Dixon MRI technique. Hepatic fat fraction (HFF), which represents hepatic fat content, was measured and calculated. ${ }^{17}$ Patients with HFF of $5.5 \%-10.0 \%, 10.1 \%$ $25.0 \%$, and $>25.0 \%$ were diagnosed as having mild, moderate, and severe fatty liver, respectively. ${ }^{18}$ Then, the patients were classified into 3 groups according to HFF: T2DM without fatty liver $(\mathrm{n}=41, \mathrm{HFF} \leq 5 \%)$, T2DM with mild fatty liver $(\mathrm{n}=34,5 \%<\mathrm{HFF} \leq 10 \%)$, and T2DM with moderate-to-severe fatty liver $(\mathrm{n}=35$, HFF $>10 \%)$, since the number of patients with moderate or severe fatty liver was relatively small. Exclusion criteria included diabetes mellitus of other types such as gestational diabetes mellitus and type 1 diabetes mellitus, hyperglycemic hyperosmolar state or diabetic ketoacidosis, infection or inflammation, diseases known to cause fatty liver such as viral hepatitis or autoimmune liver disease, taking medication known to affect the liver function or lipid metabolism for at least 3 months before the start of this study, drinking at least $140 \mathrm{~g}$ or $70 \mathrm{~g}$ of alcohol weekly for males and females, respectively, and those with a mental disorder, malignancy, or severe liver and kidney dysfunction history.

\section{Anthropometric and Biochemical Measurements}

All patients underwent physical examination, including weight, height, hip circumference (HC), waist circumference (WC) as well as blood pressure (BP) measurements. The waist/hip ratio (WHR) was determined by dividing WC by HC, whereas body mass index (BMI) was determined by dividing body weight $(\mathrm{kg})$ by squared height $\left(\mathrm{m}^{2}\right)$. Fasting peripheral blood was sampled in each case and analyzed with an automatic biochemical analyzer (HITACHI 7600, HITACHI Company, Japan) for fasting plasma glucose (FPG), high-sensitivity C-reactive protein 
(hs-CRP), aspartate aminotransferase (AST), alanine aminotransferase (ALT), serum uric acid (SUA), triglyceride (TG), total cholesterol (TC), and high/low-density lipoprotein cholesterol (HDL-c/LDL-c). An automatic immunoassay analyzer (AIA-2000ST, TOSOH company, Japan) was used to assess fasting insulin (FINS). Hemoglobin A1c (HbA1c) was measured using an automatic glycosylated hemoglobin analyzer (HLC-723G8, TOSOH). The standard procedures were followed to measure these parameters, and the experiments were performed at the Department of Laboratory Medicine, First Affiliated Hospital of Soochow University. The IR state was assessed by the homeostasis model assessment of IR (HOMA-IR) index, which was determined as follows: HOMA-IR $=$ FPG $(\mathrm{mmol} / \mathrm{L}) \times$ FINS $(\mu \mathrm{U} / \mathrm{mL}) / 22.5$. Serum TXNIP concentrations were measured using an enzyme-linked immunosorbent assay kit for humans (CycLex Company, Japan) per the manufacturer's protocols. In every case, we analyzed serum samples twice to obtain the mean TXNIP level. Typically, the serum TXNIP level was expected to be $350 \mathrm{pg} / \mathrm{mL}$, whereas a margin error of $8 \mathrm{pg} / \mathrm{mL}$ was allowed.

\section{Dixon MRI for Hepatic Fat Measurements}

All participants fasted for at least $4 \mathrm{~h}$; then, they lied in a supine position to undergo MRI examinations, where the standard torso phased-array coil was concentrated on the liver at 1.5 T (SuperVan, Lonwin Medical System, China) with an eight-channel receiver coil. The scanning protocol involved taking an initial set of localizer images and then axial images using a multiecho liver-interpolated volumeexcitation sequence with the following parameters: three echoes with the times of $2.25,3.37$, and $4.5 \mathrm{~ms}$, respectively, a flip angle of $12^{\circ}, 40$ slices with a thickness of $2.5 \mathrm{~mm}$, a matrix of $256 \times 205 \mathrm{~mm}$, a field of view of $400 \times 320 \mathrm{~mm}$, and total acquisition time of $38 \mathrm{~s}$ (first a $19 \mathrm{~s}$ scan in a free-breathing state followed by a 19 $\mathrm{s}$ scan in a breath-holding state). All subjects were trained to hold their breath during the last inspiration to ensure consistency among the subjects. MRI-HFF maps were automatically generated by a plug-in algorithm that runs on the WinStation software (WinStation, Lonwin Medical System, China). The images from the slice level $1 \mathrm{~cm}$ above the porta hepatis were reviewed by a blinded, experienced radiologist using WinStation. Then, circular regions of interest (ROIs) were delineated manually from the MRI-HFF maps in 3 liver segments for every participant. The radius of all ROIs was $1 \mathrm{~cm}$, and they were close to the segment center but were away from artifacts, liver edges, and main blood vessels. For 3 ROIs, we measured HFF values and determined the average from the 3 ROIs.

\section{Statistical Analysis}

SPSS22.0 (IBM, USA) was used for statistical analysis. Enumeration data were displayed in percentage form, and their distribution normality was analyzed by KolmogorovSmirnov test. Variables with normal distribution were presented as the mean \pm standard deviation (SD), whereas those with abnormal distribution as the median (25th, 75 th percentiles). Categorical data were evaluated by the chi-square test. Kruskal-Wallis test or one-way analysis of variance (ANOVA) test was performed for comparing continuous data across the 3 groups. Furthermore, all subjects were stratified into four groups per TXNIP quartiles. The rates or proportions among different groups were evaluated by Linear-by-Linear Association. For variables with normal distribution, linear polynomial contrasts in ANOVA were used to assess continuous variables among diverse groups, whereas those with abnormal distribution were analyzed by the Jonckheere-Terpstra test. The relationship of TXNIP with HFF was assessed by the Spearman correlation. The independent relationship between TXNIP and fatty liver to varying degrees in patients with T2DM was assessed by multinomial logistic regression. NAFLD discrimination was tested by plotting the receiver operating characteristic (ROC) curve and calculating the area under the curve (AUC) value. We determined the best threshold of TXNIP in discriminating NAFLD by Youden's index. The two-sided $P<0.05$ stood for statistical significance.

\section{Results}

Table 1 presents the clinical features of the patients. Among the 110 newly diagnosed patients with T2DM enrolled in this study, 41 were classified as without fatty liver, whereas 34 and 35 were with mild and moderate-tosevere fatty liver, respectively. The mean $\pm \mathrm{SD}$ age of the enrolled participants was $53.88 \pm 13.10$ years, and the participants had the mean $\pm \mathrm{SD}$ fasting glucose of 8.90 $\pm 2.49 \mathrm{mmol} / \mathrm{L}$ and $\mathrm{HbAlc}$ of $9.86 \% \pm 1.73 \%$. No significant differences in age, gender, BP, HbA1c, LDL-c, or TC were detected among the 3 groups. BMI, WC, $\mathrm{HC}$, and serum TXNIP levels showed significant differences among different groups, and they showed progressive increases as fatty liver levels increased. Moreover, the level of HDL-c decreased as the level of the fatty liver increased. The 
Table I Clinical and Laboratory Data in Participants Grouped by Fatty Liver Degree

\begin{tabular}{|c|c|c|c|c|c|}
\hline Variables & $\begin{array}{l}\text { All Subjects } \\
(n=I \mid 0)\end{array}$ & $\begin{array}{l}\text { Without Fatty liver } \\
(n=4 I)\end{array}$ & $\begin{array}{l}\text { Mild Fatty Liver } \\
(n=34)\end{array}$ & $\begin{array}{l}\text { Moderate to Severe Fatty } \\
\text { Liver } \\
(n=35)\end{array}$ & $P$ value \\
\hline Male sex (\%) & 53.6 & 51.2 & 61.8 & 48.6 & 0.506 \\
\hline Age (years) & $53.88 \pm 13.10$ & $52.76 \pm 11.88$ & $55.24 \pm 13.20$ & $53.89 \pm 14.55$ & 0.910 \\
\hline Hypertension (\%) & 31.8 & 31.7 & 29.4 & 34.3 & 0.908 \\
\hline HFF (\%) & $10.26 \pm 6.97$ & $4.61 \pm 0.27$ & $7.77 \pm 1.19 * *$ & $19.30 \pm 5.06 * * \#$ & $<0.001$ \\
\hline BMI $\left(\mathrm{kg} / \mathrm{m}^{2}\right)$ & $25.06 \pm 4.96$ & $21.47 \pm 2.10$ & $24.89 \pm 3.23 * *$ & $29.44 \pm 5.32 * * \#$ & $<0.001$ \\
\hline$W C(\mathrm{~cm})$ & $90.44 \pm 12.04$ & $82.61 \pm 9.65$ & $89.21 \pm 6.92 * *$ & $100.81 \pm 11.07 * * \#$ & $<0.001$ \\
\hline $\mathrm{HC}(\mathrm{cm})$ & $95.80 \pm 9.81$ & $90.05 \pm 8.90$ & $96.29 \pm 6.26 * *$ & $102.06 \pm 9.82 * * \#$ & $<0.001$ \\
\hline WHR & $0.94 \pm 0.06$ & $0.91 \pm 0.04$ & $0.93 \pm 0.04$ & $0.99 \pm 0.06 * * \#$ & $<0.001$ \\
\hline HbAlc (\%) & $9.86 \pm 1.73$ & $10.01 \pm 1.85$ & $9.88 \pm 1.96$ & $9.65 \pm 1.34$ & 0.676 \\
\hline FPG $(\mathrm{mmol} / \mathrm{L})$ & $8.90 \pm 2.49$ & $8.36 \pm 2.62$ & $8.27 \pm 1.96$ & $10.17 \pm 2.36 * * \#$ & 0.001 \\
\hline FINS $(\mu \mathrm{U} / \mathrm{mL})$ & $13.88 \pm 7.48$ & 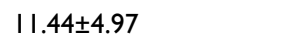 & $13.01 \pm 6.60$ & $17.57 \pm 9.27 * * \#$ & 0.001 \\
\hline HOMA-IR & $5.57 \pm 3.42$ & $4.34 \pm 2.35$ & $4.80 \pm 2.55$ & $7.75 \pm 4.18^{* * \# \#}$ & $<0.001$ \\
\hline ALT (U/L) & $21.50(15.50,34.78)$ & $17.10(14.25,22.15)$ & $18.80(\mid 4.28,25.35)$ & $38.70(27.80,53.60)^{* * \# \#}$ & $<0.001$ \\
\hline AST (U/L) & $20.65(I 5.58,27.83)$ & $17.50(14.65,21.85)$ & $18.65(14.98,21.55)$ & $30.60(22.30,43.50)^{* * \# \#}$ & $<0.001$ \\
\hline SUA $(\mu \mathrm{mol} / \mathrm{L})$ & $319.55 \pm 88.75$ & $292.00 \pm 67.31$ & $304.20 \pm 85.30$ & $366.73 \pm 97.05 * * \#$ & $<0.001$ \\
\hline $\mathrm{TC}(\mathrm{mmol} / \mathrm{L})$ & $4.72 \pm 0.95$ & $4.56 \pm 0.88$ & $4.69 \pm 1.11$ & $4.93 \pm 0.86$ & 0.229 \\
\hline TG (mmol/L) & $1.35(0.98,2.16)$ & $1.05(0.77,1.24)$ & $\mathrm{I} .3 \mathrm{I}(0.9 \mathrm{I}, \mathrm{I} .8 \mathrm{I})$ & $2.30(1.53,3.66)^{* * \# \#}$ & $<0.001$ \\
\hline HDL-c (mmol/L) & $\mid .22 \pm 0.31$ & $1.38 \pm 0.28$ & $1.22 \pm 0.29 *$ & $1.04 \pm 0.26 * * \#$ & $<0.001$ \\
\hline LDL-c (mmol/L) & $2.63 \pm 0.73$ & $2.55 \pm 0.66$ & $2.55 \pm 0.85$ & $2.8 I \pm 0.68$ & 0.222 \\
\hline hs-CRP (mg/L) & $1.74(0.72,3.69)$ & $0.92(0.47,2.43)$ & $1.5 \mathrm{I}(0.58,2.17)$ & $3.45(1.90,8.95)^{* * \# \#}$ & $<0.001$ \\
\hline TXNIP (pg/mL) & $350.06 \pm 85.88$ & $293.86 \pm 67.59$ & $363.78 \pm 76.23 * *$ & $402.57 \pm 76.13^{* * \# \#}$ & $<0.001$ \\
\hline
\end{tabular}

Notes: ${ }^{*} P<0.05$, and ${ }^{* *} P<0.01$ vs Without fatty liver group; ${ }^{\#} P<0.01$ vs Mild fatty liver group.

patients with diabetes and moderate-to-severe fatty liver had higher WHR, FPG, FINS, HOMA-IR, ALT, AST, SUA, TG, and hs-CRP than the patients with diabetes with mild fatty liver or without fatty liver (Table 1).

Next, all subjects were stratified in accordance with the quartiles of serum TXNIP (Quartile 1 (Q1): $\leq 290.99 \mathrm{pg} / \mathrm{mL}$, Quartile 2 (Q2): 290.99-348.71 pg/mL, Quartile 3 (Q3): 348.71-413.70 pg/mL, and Quartile 4 (Q4): $>413.70 \mathrm{pg} /$ $\mathrm{mL}$ ). As shown in Figure 1, in the subjects in high TXNIP quartiles, BMI, WC, FPG, SUA, and TG were increased, whereas HDL-c was significantly decreased. In general, the prevalence of severe fatty liver showed an increasing trend as the TXNIP quartiles increased (for all trends, $P<0.05$ ) (Figure 2). For example, moderate-to-severe fatty liver incidence rates were $2.7 \%$ in Q1, 5.5\% in Q2, $10.9 \%$ in Q3, and $12.7 \%$ in Q4. Furthermore, upon Spearman correlation analysis, serum TXNIP level was significantly related to fatty liver level (HFF) $(\mathrm{r}=0.516, P<0.001)$.

When non-NAFLD diabetics were used as controls in the multinomial logistic regression model, TXNIP was significantly correlated with severe fatty liver prevalence (odds ratio $(\mathrm{OR})=1.014, P<0.001$ for mild fatty liver; $\mathrm{OR}=$ $1.022, P<0.001$ for moderate-to-severe fatty liver) when sex and age were adjusted (Table 2). After the further adjustment of FPG, FINS, TC, TG, HDL-c, and LDL-c, serum TXNIP was significantly related to fatty liver level $(\mathrm{OR}=1.014, P=$ 0.002 for mild fatty liver; $\mathrm{OR}=1.019, P=0.001$ for moderateto-severe fatty liver). TXNIP significantly affected severe fatty liver when BMI, WC, SUA, and hs-CRP were adjusted (OR = $1.012, P=0.014$ for mild fatty liver; $\mathrm{OR}=1.013, P=0.039$ for moderate-to-severe fatty liver) (Table 2 ).

ROC curves were constructed to evaluate whether TXNIP was a biomarker used to diagnose NAFLD among diabetics. The results showed that TXNIP was a significant predictor of the Dixon MRI-measured fatty liver with the AUC value of 0.811 (95\% confidence interval (CI): 0.729-0.893, $P<0.001)$. After adjustment for age and sex, the AUC value was 0.820 (95\% CI: $0.739-0.900$, $P<0.001)$. The optimal threshold of TXNIP in discriminating NAFLD was determined to be $\geq 326.87 \mathrm{ng} / \mathrm{mL}$ $(81.2 \%$ and $75.6 \%$ for sensitivity and specificity, respectively) (Figure 3).

\section{Discussion}

TXNIP plays a key role in lipid/glucose metabolism. Though many studies have focused on the effect of 

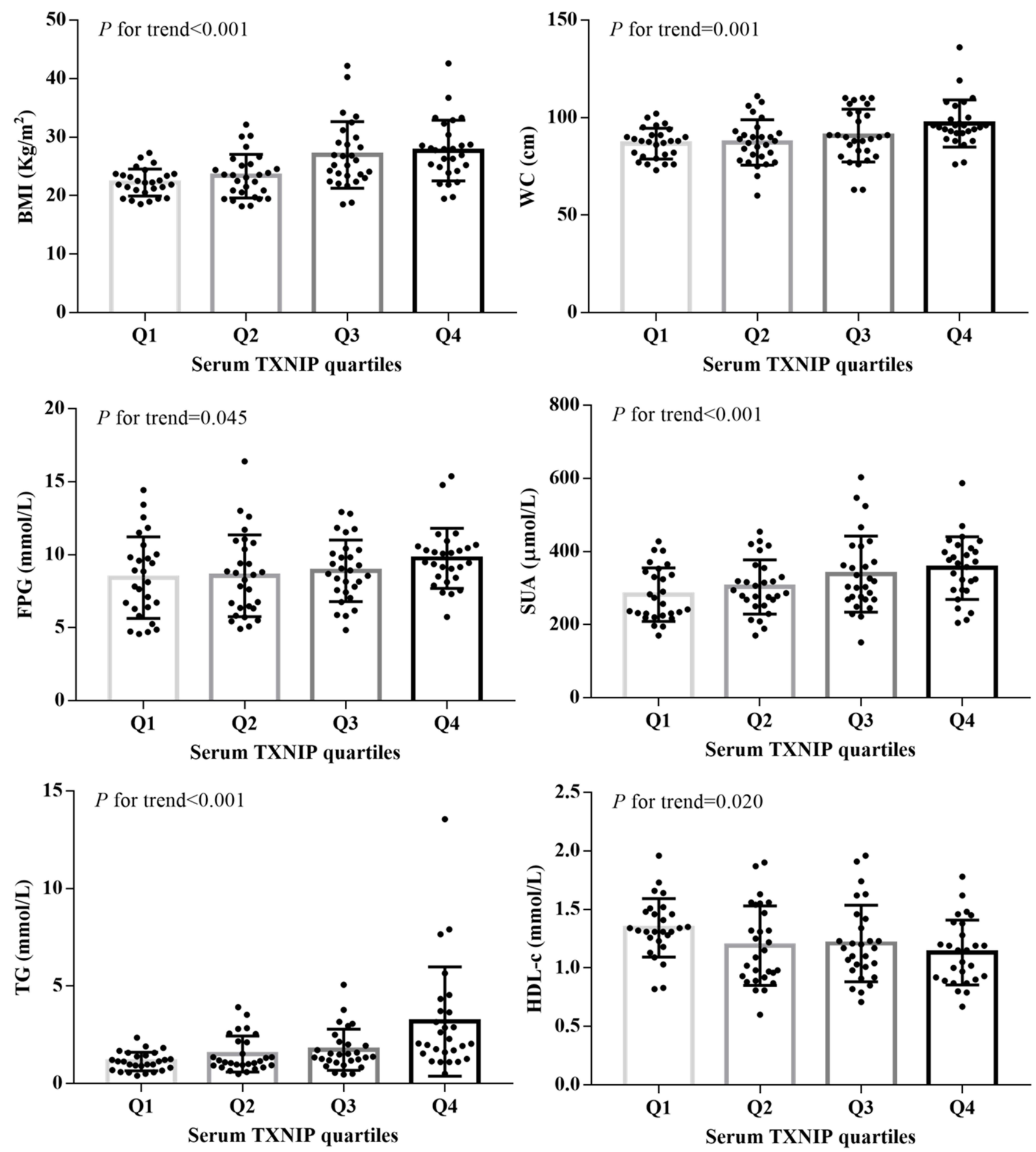

Figure I The main characteristics of study participants by quartiles of serum TXNIP. Data are the mean \pm SD.

TXNIP on fatty liver formation, a few studies have reported the relationship between serum TNXIP and NAFLD. To the best of our knowledge, our work is the first to analyze the association of circulating TXNIP with Dixon MRI-measured fatty liver in patients with T2DM. In this study, we selected patients with newly diagnosed
T2DM to exclude the effect of anti-diabetic drugs, such as metformin, insulin, and glucagon-like peptide-1, on liver fat content. ${ }^{19,20}$ First, circulating TXNIP levels among the patients with T2DM with NAFLD remarkably increased compared with those in the diabetic patients without fatty liver. Next, all patients were stratified per the quartiles of 


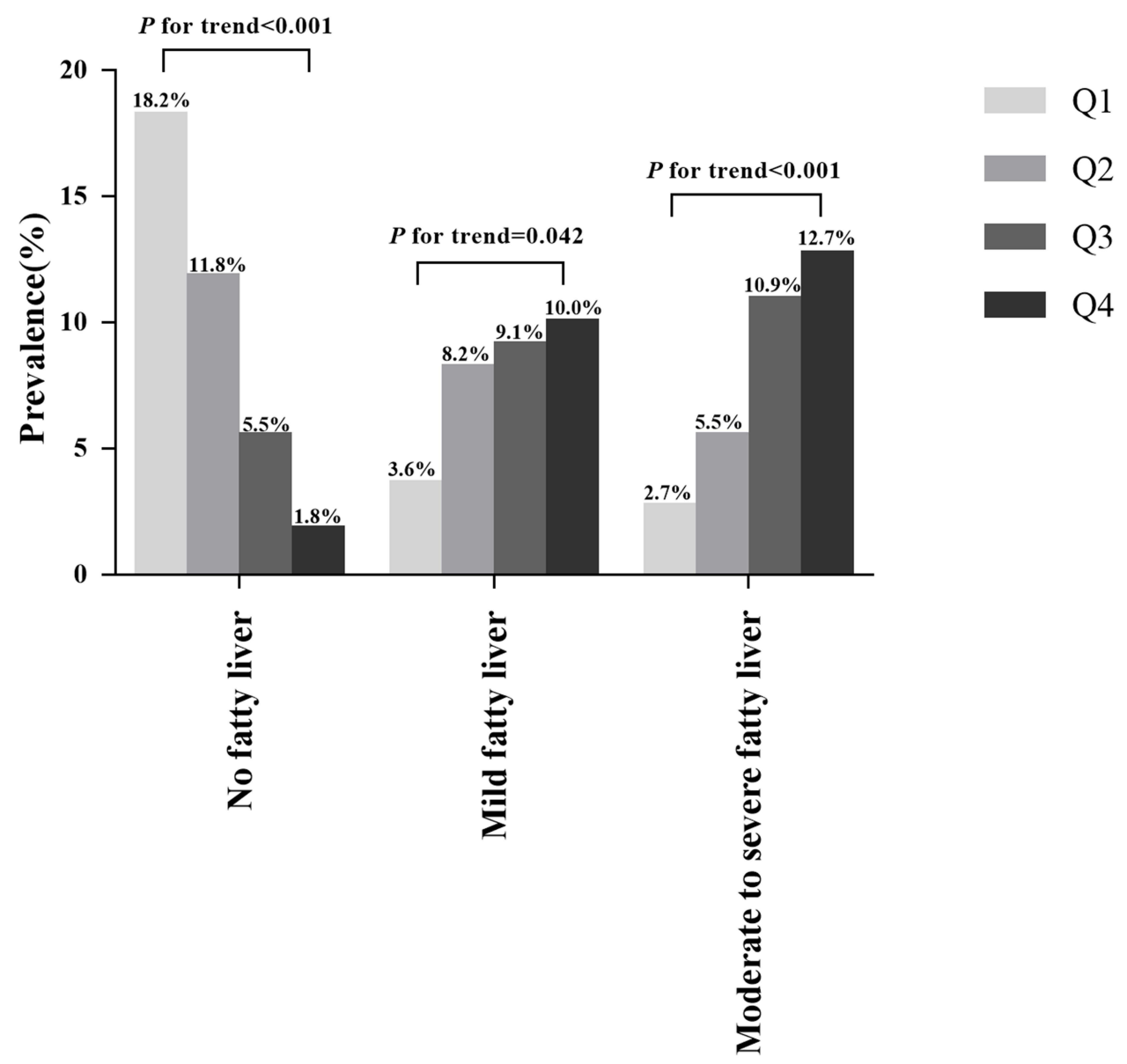

Figure 2 Prevalence of fatty liver by severity, as a function of TXNIP quartile.TXNIP quartile: QI: $\leq 290.99 \mathrm{pg} / \mathrm{mL}, \mathrm{Q} 2: 290.99 \sim 348.71 \mathrm{pg} / \mathrm{mL}, \mathrm{Q} 3: 348.71 \sim 413.70 \mathrm{pg} / \mathrm{mL}$, Q4: $>413.70 \mathrm{pg} / \mathrm{mL}$.

TXNIP, and the results showed that the prevalence of severe NAFLD in the patients with T2DM increased with the ascending quartiles of TXNIP. In addition, TXNIP was positively correlated with HFF, and further multinomial logistic regression analysis revealed that TXNIP significantly affected fatty liver with any level of severity. Then, the ROC curve results showed that TXNIP was a significant predictor of NAFLD in patients with T2DM. However, as this was a cross-sectional study, we could not determine the causality between TXNIP and NAFLD. Therefore, the associations concluded in this study should be cautiously interpreted.

Previous basic studies have shown that TXNIP may be an upstream regulator in the development of hepatic steatosis. First, TXNIP aggravates hepatic oxidative stress; thus, causing increased lipid synthesis and deposition in the liver. Excess lipid accumulation induces inflammatory responses, which further accelerate hepatic lipid accumulation and ultimately lead to liver injury. ${ }^{21,22}$ Second,
TXNIP directly mediates liver inflammation through NLRP3 inflammasomes, followed by the release of various inflammatory cytokines; thus, promoting lipid deposition in hepatocytes. ${ }^{23}$ Third, TXNIP attenuates the insulin sensitivity of peripheral tissues; thus, increasing hepatic glucose absorption and subsequently activating intracellular glycolysis induced by carbohydrate response elementbinding protein, which provides metabolic precursor materials for further hepatic lipogenesis. ${ }^{24}$ Finally, TXNIP weakens lipolysis inhibition regulated by insulin within adipose tissues, which releases many free fatty acids (FFAs) in circulation. The increased FFA levels in circulation act as the main source of nonesterified fatty acids for TC produced within hepatocytes; thus, causing increased lipid synthesis and deposition in the liver. ${ }^{8}$ This study suggested that circulating TXNIP was significantly related to the severity of fatty liver, which further explained that TXNIP has a key role in modulating the metabolism of liver lipids. 
Table 2 Associations Between TXNIP and Different Degrees of Fatty Liver After Controlling for Confounding Factors

\begin{tabular}{|l|l|l|l|l|}
\hline \multirow{2}{*}{} & \multicolumn{2}{l|}{ T2DM with Mild Fatty Liver } & \multicolumn{2}{l|}{ T2DM with Moderate to Severe Fatty Liver } \\
\cline { 2 - 5 } & OR (95\% Cl) & P value & OR (95\% CI) & P value \\
\hline Model 1 & $1.013(1.006-1.021)$ & $<0.001$ & $1.021(1.012-1.030)$ & $<0.001$ \\
Model 2 & $1.014(1.007-1.022)$ & $<0.001$ & $1.022(1.013-1.031)$ & $<0.001$ \\
Model 3 & $1.014(1.005-1.024)$ & 0.002 & $1.019(1.008-1.031)$ & 0.001 \\
Model 4 & $1.012(1.002-1.022)$ & 0.014 & $1.013(1.001-1.026)$ & 0.039 \\
\hline
\end{tabular}

Notes: Model I: crude. Model 2: adjusted for age and sex. Model 3: adjusted for Model 2 + FPG, FINS, TC, TG, HDL-c, LDL-c. Model 4: adjusted for Model 3 + BMI, WC, SUA, hs-CRP.

This study has several limitations. First, as this was a cross-sectional study, we could not conclude causes and effects. Second, this study had a small sample size, which might affect the statistical accuracy of the results. More large-scale longitudinal studies should be performed to establish the cause-effect relation. Third, lipid levels were detected according to the Dixon MRI results but not liver biopsy results. At present, liver biopsy is a gold standard in the diagnosis of NAFLD. Nonetheless, it is an invasive approach that may result in great risks such as postprocedural infection, and it is rarely performed among normal subjects with no clinical indication. Therefore, the imaging approaches such as magnetic resonance spectroscopy, computed tomography, ultrasonography, and Dixon MRI provide noninvasive means for quantifying intrahepatic lipid content accurately. ${ }^{25}$ Among these imaging methods, the two-point DIXON technique can rapidly generate high-resolution separated fat-alone and wateralone liver images. In nonhomogeneous liver tissues, DIXON-based liver fat quantification is highly accurate compared with standard liver fat histopathological evaluation; as a result, it has been used more to quantify liver fat. ${ }^{26}$ However, Dixon MRI underestimates patients suffering from moderate and severe steatosis, such as fibrosis and necroinflammation.

To conclude, the present findings provide novel evidence showing that circulating TXNIP level is significantly correlated with NAFLD severity in patients with T2DM, which offers an efficient way to evaluate and monitor fatty liver occurrence and development in diabetics. Our results suggested that TXNIP is a biomarker, and more clinical trials and prospective studies should be performed to interpret the role of TXNIP in NAFLD occurrence and progression among patients with T2DM.

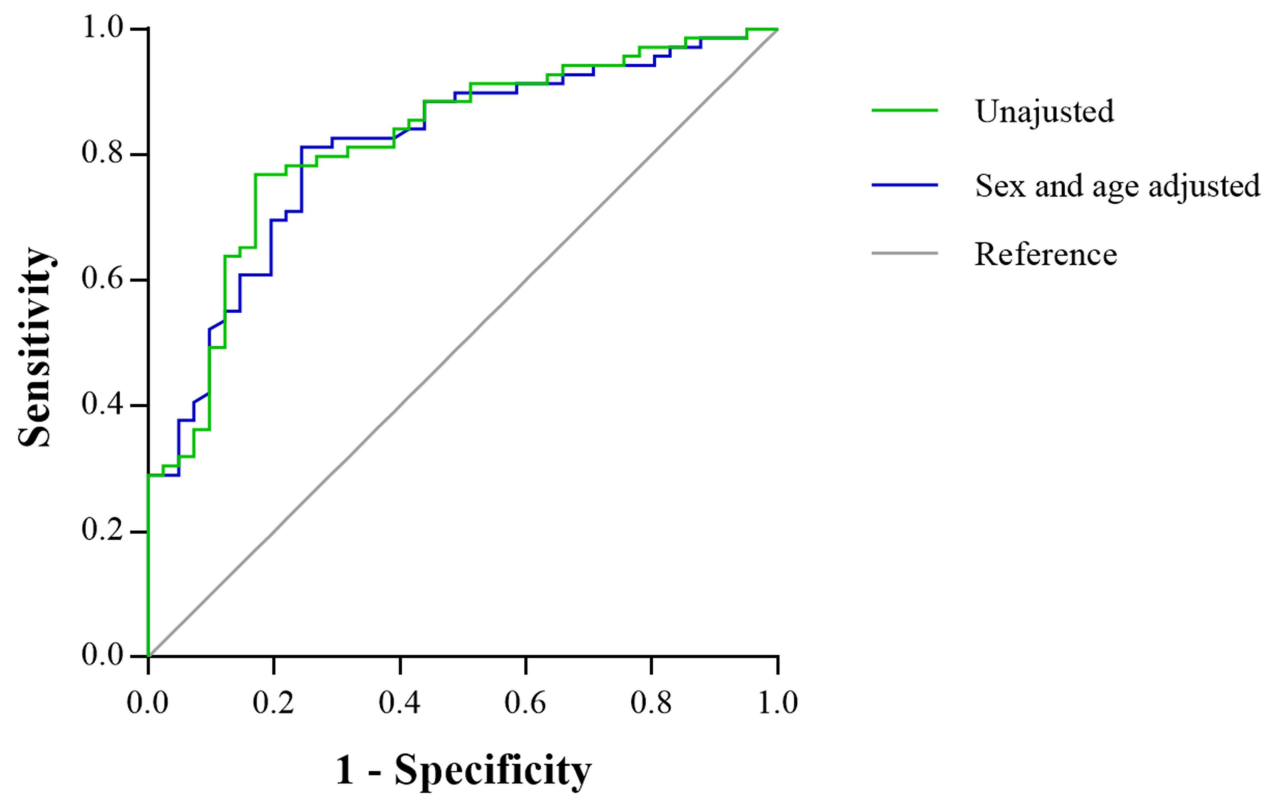

Figure 3 Receiver operating characteristic (ROC) curves for TXNIP and in patients with T2DM. Unadjusted: AUC=0.8I I $(95 \%$ Cl: $0.729 \sim 0.893, P<0.00 \mathrm{I})$; Sex and age adjusted: $A \cup C=0.820$ (95\% Cl: 0.739 0.900, P<0.00I). 
Furthermore, fundamental studies are needed for discovering possible mechanisms underlying TXNIP-regulated NAFLD.

\section{Abbreviations}

TXNIP, thioredoxin-interacting protein; NAFLD, nonalcoholic fatty liver disease; T2DM, type 2 diabetes mellitus; MRI, magnetic resonance imaging; HFF, hepatic fat fraction; NASH, nonalcoholic steatohepatitis; HCC, hepatocellular carcinoma; IR, insulin resistance; GDM, gestational diabetes mellitus; T1DM, type 1 diabetes mellitus; HC, hip circumference; WC, waist circumference; BP, blood pressure; WHR, waist/hip ratio; BMI, body mass index; FPG, fasting plasma glucose; hs-CRP, highsensitivity C-reactive protein; AST, aspartate aminotransferase; ALT, alanine aminotransferase; SUA, serum uric acid; TG, triglyceride; TC, total cholesterol; HDL-c/LDLc, high/low-density lipoprotein cholesterol; FFAs, free fatty acids; CT, computed tomography.

\section{Ethics Statements}

The study protocol was approved by the Ethics Committee of the First Affiliated Hospital of Soochow University in accordance with the principles of the Helsinki Declaration and written informed consent was obtained from each subject.

\section{Acknowledgments}

This work was supported by grants from People's Livelihood Science and Technology of Suzhou (grant no. SYS2020104 to Hong Sun, no. SS2020008 to Bimin Shi), National Natural Science Youth Foundation of China (grant no. 81700632 to Hong Sun), the Natural Science Youth Foundation of Jiangsu Province (grant no. BK20170366 to Hong Sun), and China Postdoctoral Science Foundation (2020M671559 to Juan Chen). Yuting Guo and Juan Chen are co-first authors for this study.

\section{Disclosure}

The authors have declared that no competing interest exists.

\section{References}

1. Zhou JH, Cai JJ, She ZG, Li HL. Noninvasive evaluation of nonalcoholic fatty liver disease: current evidence and practice. World J Gastroenterol. 2019;25(11):1307-1326. doi:10.3748/wjg.v25.i11.1307

2. Tilg H, Moschen AR, Roden M. NAFLD and diabetes mellitus. Nat Rev Gastroenterol Hepatol. 2017;14(1):32-42. doi:10.1038/nrga stro.2016.147
3. Lomonaco R, Bril F, Portillo-Sanchez P, et al. Metabolic impact of nonalcoholic steatohepatitis in obese patients with type 2 diabetes. Diabetes Care. 2016;39(4):632-638. doi:10.2337/dc15-1876

4. Li L, Yang X. The essential element manganese, oxidative stress, and metabolic diseases: links and interactions. Oxid Med Cell Longev. 2018;2018:7580707. doi:10.1155/2018/7580707

5. Day CP, James OF. Steatohepatitis: a tale of two "hits"? Gastroenterology. 1998;114(4):842-845. doi:10.1016/S0016-5085 (98)70599-2

6. Tilg H, Moschen AR. Evolution of inflammation in nonalcoholic fatty liver disease: the multiple parallel hits hypothesis. Hepatology. 2010;52(5):1836-1846. doi:10.1002/hep.24001

7. Hu J, Yu Y. The function of thioredoxin-binding protein-2 (TBP-2) in different diseases. Oxid Med Cell Longev. 2018;2018:4582130. doi:10.1155/2018/4582130

8. Alhawiti NM, Al Mahri S, Aziz MA, Malik SS, Mohammad S. TXNIP in metabolic regulation: physiological role and therapeutic outlook. Curr Drug Targets. 2017;18(9):1095-1103. doi:10.2174/ 1389450118666170130145514

9. Ferreira NE, Omae S, Pereira A, et al. Thioredoxin interacting protein genetic variation is associated with diabetes and hypertension in the Brazilian general population. Atherosclerosis. 2012;221(1):131-136. doi:10.1016/j.atherosclerosis.2011.12.009

10. Szpigel A, Hainault I, Carlier A, et al. Lipid environment induces ER stress, TXNIP expression and inflammation in immune cells of individuals with type 2 diabetes. Diabetologia. 2018;61(2):399-412. doi:10.1007/s00125-017-4462-5

11. Anderson EJ. Cutting calories and TXNIP from the skeletal muscle to restore insulin sensitivity. Diabetes. 2016;65(1):16-18. doi:10.2337/ dbi15-0021

12. Gao Y, Chen S, Peng M, et al. Correlation between thioredoxin-interacting protein and nerve conduction velocity in patients with type 2 diabetes mellitus. Front Neurol. 2020;11:733. doi:10.3389/fneur.2020.00733

13. Xu LL, Gao W, Chen ZM, et al. Relationships between diabetic nephropathy and insulin resistance, inflammation, Trx, Txnip, CysC and serum complement levels. Eur Rev Med Pharmacol Sci. 2020;24 (22):11700-11706. doi:10.26355/eurrev_202011_23815

14. Park HS, Song JW, Park JH, et al. TXNIP/VDUP1 attenuates steatohepatitis via autophagy and fatty acid oxidation. Autophagy. 2020;17:1-16.

15. Zhang X, Zhang JH, Chen XY, et al. Reactive oxygen species-induced TXNIP drives fructose-mediated hepatic inflammation and lipid accumulation through NLRP3 inflammasome activation. Antioxid Redox Signal. 2015;22(10):848-870. doi:10.1089/ars.2014.5868

16. Mohamed IN, Sarhan NR, Eladl MA, El-Remessy AB, El-Sherbiny M. Deletion of Thioredoxin-interacting protein ameliorates high fat diet-induced non-alcoholic steatohepatitis through modulation of Toll-like receptor 2-NLRP3-inflammasome axis: histological and immunohistochemical study. Acta Histochem. 2018;120(3):242-254. doi:10.1016/j.acthis.2018.02.006

17. Tang A, Desai A, Hamilton G, et al. Accuracy of MR imaging-estimated proton density fat fraction for classification of dichotomized histologic steatosis grades in nonalcoholic fatty liver disease. Radiology. 2015;274 (2):416-425. doi:10.1148/radiol.14140754

18. Caussy C, Brissot J, Singh S, et al. Prospective, same-day, direct comparison of CAP with the M vs the XL probe in patients with nonalcoholic fatty liver disease, using magnetic resonance imaging proton density fat fraction as the standard. Clin Gastroenterol Hepatol. 2020;18(8):1842-1850.e6. doi:10.1016/j.cgh.2019.11.060

19. Pinyopornpanish K, Leerapun A, Chattipakorn N, Chattipakorn N. Effects of metformin on hepatic steatosis in adults with nonalcoholic fatty liver disease and diabetes: insights from the cellular to patient levels. Gut Liver. 2021;15(6):827-840. doi:10.5009/ gn120367 
20. Guo W, Tian W, Lin L, Xu X. Liraglutide or insulin glargine treatments improves hepatic fat in obese patients with type 2 diabetes and nonalcoholic fatty liver disease in twenty-six weeks: a randomized placebo-controlled trial. Diabetes Res Clin Pract. 2020;170:108487. doi:10.1016/j.diabres.2020.108487

21. Kawamoto E, Tamakoshi K, Ra SG, Masuda H, Kawanaka K. Immobilization rapidly induces thioredoxin-interacting protein gene expression together with insulin resistance in rat skeletal muscle. $J$ Appl Physiol. 2018;125(2):596-604. doi:10.1152/japplphysiol.00951.2017

22. Ahn B, Soundarapandian MM, Sessions H, et al. MondoA coordinately regulates skeletal myocyte lipid homeostasis and insulin signaling. J Clin Invest. 2016;126(9):3567-3579. doi:10.1172/JCI87382

23. Zhou F, Zhang Y, Chen J, Hu Y, Xu Y. Verapamil ameliorates hepatic metaflammation by inhibiting thioredoxin-interacting protein/NLRP3 pathways. Front Endocrinol (Lausanne). 2018;9:640. doi:10.3389/ fendo.2018.00640
24. Saponaro C, Gaggini M, Gastaldelli A. Nonalcoholic fatty liver disease and type 2 diabetes: common pathophysiologic mechanisms. Curr Diab Rep. 2015;15(6):607. doi:10.1007/s11892-015-0607-4

25. Kramer H, Pickhardt PJ, Kliewer MA, et al. Accuracy of liver fat quantification with advanced CT, MRI, and ultrasound techniques: prospective comparison with MR spectroscopy. AJR Am J Roentgenol. 2017;208(1):92-100. doi:10.2214/AJR.16.16565

26. Hu F, Yang R, Huang Z, et al. 3D Multi-Echo Dixon technique for simultaneous assessment of liver steatosis and iron overload in patients with chronic liver diseases: a feasibility study. Quant Imaging Med Surg. 2019;9(6):1014-1024. doi:10.21037/ qims.2019.05.20

\section{Publish your work in this journal}

Diabetes, Metabolic Syndrome and Obesity: Targets and Therapy is an international, peer-reviewed open-access journal committed to the rapid publication of the latest laboratory and clinical findings in the fields of diabetes, metabolic syndrome and obesity research. Original research, review, case reports, hypothesis formation, expert opinion and commentaries are all considered for publication. The manuscript management system is completely online and includes a very quick and fair peer-review system, which is all easy to use. Visit http://www.dovepress.com/testimonials.php to read real quotes from published authors. 